\title{
Some product formulas for theta functions in one and two variables
}

by

\author{
David Grant (Boulder, CO)
}

Dedicated to Harold Stark on the occasion of his 60th birthday

Introduction. For $\tau \in \mathfrak{h}=\{x+i y \mid y>0\}, a, b \in \mathbb{R}$, we define the theta function in one variable $z \in \mathbb{C}$ with characteristic vector $\left[\begin{array}{l}a \\ b\end{array}\right]$ by

$$
\theta\left[\begin{array}{l}
a \\
b
\end{array}\right](z, \tau)=\sum_{n \in \mathbb{Z}} e^{\pi i(n+a)^{2} \tau+2 \pi i(n+a)(z+b)} .
$$

Let $m$ be a positive integer. The following equation between modular forms is crucial to the construction of elliptic units:

$$
\prod_{\substack{0 \leq u, v<m \\
(u, v) \neq(0,0)}} \theta\left[\begin{array}{l}
1 / 2+u / m \\
1 / 2+v / m
\end{array}\right](0, \tau)=(-1)^{m-1} m \eta(\tau)^{m^{2}-1},
$$

where $\eta(\tau)=e^{\pi i \tau / 12} \prod_{n>0}\left(1-e^{2 \pi i n \tau}\right)$. For example, Stark (see [S, p. 353]) proved (1) (in a disguised form) using Kronecker's second limit formula, a tool which is not available for the study of theta functions in more than 1 variable.

Note that $\eta(\tau)^{24}=\Delta(\tau)$, a cusp form of weight 12 related to the discriminant of the elliptic curve which has $\tau$ as a period. No formula analogous to (1) holds for all $m$ relating theta functions in two variables to the "discriminant modular form" attached to $\tau$ in the Siegel upper half-space of degree 2 (see §3). One main purpose of this paper is to prove Theorem 2 of Section 3, which shows that an analogous formula does hold when $m=3$ and $m=4$.

That such an equation holds with an undetermined constant was shown for $m=3$ in [Gr4], and independently for $m=3$ and $m=4$ by Goren [Go]. Our approach is different from that in [Go], using facts about Siegel modular forms, rather than considering the moduli of genus two curves. At

2000 Mathematics Subject Classification: 11F46, 11G16, $14 \mathrm{~K} 25$. 
the end of Section 3 we briefly discuss how the two approaches compare, relating the product formulas of Theorem 2 to the fact that primitive 3and 4-torsion points on the Jacobian of a curve of genus 2 do not lie on the embedded image of the curve under the Albanese map using a Weierstrass point as base point. This fact is also central to the arguments in [BoBa], $[\mathrm{BaBo}]$, and [Gr1], which for certain genus 2 curves defined over number fields, build units in number fields attached to torsion points. See $[A]$ for similar results for genus 3 curves, and [dSG], [Gr2], [FK] and [Lec] for more on units attached to genus 2 curves. In particular, for one curve, $[\mathrm{FK}]$ builds units from 6-torsion points on the Jacobian from the point of view of theta functions. I hope that the type of product formulas given here will lead to a better understanding of the sort of norm computations done in [FK]. In some sense, Theorem 2 says that the arithmetic properties enjoyed by 3and 4-torsion points on Jacobians of curves of genus 2 defined over number fields are reflected in the geometry of generic curves of genus 2 .

Another product formula for theta functions in two variables is given in [Gr3] (see also [C]), and a seemingly unrelated product formula is given in [Bor].

The other main purpose of this paper is to derive generalizations of Jacobi's derivative formula for theta functions in one variable, relating in Theorem 1 the products of derivatives at zero of theta functions with different rational characteristics to powers of $\eta(\tau)$. This is necessary for determining the constants in Theorem 2. For more on this theme, see [BG].

For the convenience of the reader, in Section 1 we recall certain properties of theta functions in several variables. Since we need it in what follows, in Section 2 we give a quick proof of (1) and a bevy of allied formulas. We also state and prove Theorem 1. Theorem 2 is stated and proved in Section 3.

Some of this work was done while the author was supported by the National Science Foundation. I would also like to thank John Boxall for useful discussions on this material while I was enjoying the hospitality of the University of Caen.

Finally, the germ of this paper was in some work done under the supervision of Harold Stark [Gr4]. I would like to take this opportunity to thank him for his continued support and friendship, and it is a pleasure to dedicate this paper to him.

1. Properties of theta functions. Let $\mathfrak{h}_{g}$ denote the Siegel upper halfspace of degree $g$; that is, $g \times g$ symmetric complex matrices with positivedefinite imaginary part. We let $\mathrm{Sp}_{2 g}(\mathbb{Z})$ denote the integral symplectic group of degree $g$; i.e., block matrices $\left(\begin{array}{ll}A & B \\ C & D\end{array}\right)$ such that 


$$
{ }^{\mathrm{t}}\left(\begin{array}{cc}
A & B \\
C & D
\end{array}\right)\left(\begin{array}{cc}
0 & -I \\
I & 0
\end{array}\right)\left(\begin{array}{cc}
A & B \\
C & D
\end{array}\right)=\left(\begin{array}{cc}
0 & -I \\
I & 0
\end{array}\right),
$$

where $A, B, C$, and $D$ are integral $g \times g$ matrices, $I$ is the $g \times g$ identity, and ${ }^{\mathrm{t}}$ denotes the transpose. For $N>0$, we let $\Gamma(N)$ denote the subgroup of matrices congruent to the identity $\bmod N$. Elements $\gamma=\left(\begin{array}{ll}A & B \\ C & D\end{array}\right) \in \Gamma=\Gamma(1)$ act on $\mathfrak{h}_{g}$ via $\gamma \circ \tau=(A \tau+B)(C \tau+D)^{-1}$. Let $k$ be a non-negative integer. Recall that $M_{k}(\Gamma(N))$, the space of Siegel modular forms of degree $g$, level $N$, and weight $k$, consists of holomorphic functions $f$ on $\mathfrak{h}_{g}$ satisfying

$$
f(\gamma \circ \tau)=j_{\gamma}(\tau)^{k} f(\tau),
$$

for any $\gamma=\left(\begin{array}{ll}A & B \\ C & D\end{array}\right) \in \Gamma(N)$, where $j_{\gamma}(\tau)=\operatorname{det}(C \tau+D)$. When $g=1$, we also require that $f$ be analytic on the compactification of $\Gamma(N) \backslash \mathfrak{h}_{1}$ gotten by adjoining points at the cusps.

Writing $\mathbb{R}^{g}$ and $\mathbb{Z}^{g}$ as column vectors, for any $a, b \in \mathbb{R}^{g}, \tau \in \mathfrak{h}_{g}$, we let

$$
\theta\left[\begin{array}{l}
a \\
b
\end{array}\right](z, \tau)=\sum_{n \in \mathbb{Z}^{g}} e^{\pi i^{\mathrm{t}}(n+a) \tau(n+a)+2 \pi i^{\mathrm{t}}(n+a)(z+b)}
$$

denote the theta function in $g$ variables $z \in \mathbb{C}^{g}$ with characteristic vector $\left[\begin{array}{l}a \\ b\end{array}\right]$. In particular, if $a, b \in \frac{1}{2} \mathbb{Z}^{g}$, we call $\left[\begin{array}{l}a \\ b\end{array}\right]$ a theta characteristic. We call a theta characteristic even or odd depending respectively on whether $\theta\left[\begin{array}{l}a \\ b\end{array}\right](z, \tau)$ is an even or odd function of $z$, i.e., whether $e^{4 \pi i^{\mathrm{t}} a b}$ is 1 or -1 . We identify theta characteristics mod 1 . It follows immediately from (2) that

$$
\theta\left[\begin{array}{l}
a+p \\
b+q
\end{array}\right](z, \tau)=e^{2 \pi i^{\mathrm{t}} a q} \theta\left[\begin{array}{l}
a \\
b
\end{array}\right](z, \tau)
$$

for $p, q \in \mathbb{Z}^{g}$. Hence if $a, b \in \frac{1}{m} \mathbb{Z}^{g}$, then $\theta\left[\begin{array}{l}a \\ b\end{array}\right](z, \tau)^{m}$ depends only on $\left[\begin{array}{l}a \\ b\end{array}\right]$ mod 1 . Therefore we lose at most a sign when we identify theta characteristics $\bmod 1$. 182])

For any $\gamma=\left(\begin{array}{ll}A & B \\ C & D\end{array}\right) \in \Gamma$, theta functions transform as ([I2, pp. 85, 176,

4) $\theta\left[\begin{array}{l}a \\ b\end{array}\right]^{\gamma}\left({ }^{\mathrm{t}}(C \tau+D)^{-1} z, \gamma \circ \tau\right)$

$$
=\zeta(\gamma) j_{\gamma}(\tau)^{1 / 2} e^{\pi i^{\mathrm{t}} z(C \tau+D)^{-1} C z} \theta\left[\begin{array}{l}
a \\
b
\end{array}\right](z, \tau),
$$

where

$$
\begin{aligned}
& \zeta(\gamma)=\varrho(\gamma) e^{-\pi i\left[{ }^{\mathrm{t}} a^{\mathrm{t}} B D a-2^{\mathrm{t}} a^{\mathrm{t}} B C b+{ }^{\mathrm{t}} b^{\mathrm{t}} A C b-\left({ }^{\mathrm{t}} a^{\mathrm{t}} D-{ }^{\mathrm{t}} b^{\mathrm{t}} C\right)\left(A^{\mathrm{t}} B\right)_{0}\right]}, \\
& \varrho(\gamma)=\text { an eighth root of } 1(=\mathrm{a} \text { fourth root of } 1 \text { for } \gamma \in \Gamma(2)),
\end{aligned}
$$$$
\left[\begin{array}{l}
a \\
b
\end{array}\right]^{\gamma}=\left(\begin{array}{cc}
D & -C \\
-B & A
\end{array}\right)\left[\begin{array}{l}
a \\
b
\end{array}\right]+\frac{1}{2}\left[\begin{array}{c}
\left(C^{\mathrm{t}} D\right)_{0} \\
\left(A^{\mathrm{t}} B\right)_{0}
\end{array}\right]
$$ 
and where for a matrix $M,(M)_{0}$ denotes the column vector consisting of the diagonal entries of $M$, and $j_{\gamma}(\tau)^{1 / 2}$ is a choice of branch of square root of $j_{\gamma}(\tau)$.

For $\gamma \in \Gamma$, the map $\left[\begin{array}{l}a \\ b\end{array}\right] \mapsto\left[\begin{array}{l}a \\ b\end{array}\right]^{\gamma} \bmod 1$ gives an action on characteristic vectors mod 1 we call the symplectic action. It is clear that the theta characteristics are stable under the symplectic action, but it can be shown ([I2, p. 213]) that the subsets of even and odd theta characteristics are also left stable by the symplectic action.

For any positive integer $m$, and theta characteristic $\delta$, we let $\operatorname{prim}(m)$ be the set of characteristic vectors mod 1 defined by

$$
\operatorname{prim}(m)=\left\{\left[\begin{array}{l}
a \\
b
\end{array}\right] \bmod 1 \mid m a, m b \in \mathbb{Z}^{g},(m a, m b, m)=1\right\},
$$

and set

$$
\operatorname{char}_{\delta}(m)=\delta+\operatorname{prim}(m) \bmod 1 .
$$

Note that if $\sigma_{g}(m)$ is the cardinality of $\operatorname{prim}(m)$, then

$$
\sigma_{g}(m)=m^{2 g} \prod_{p \mid m}\left(1-\frac{1}{p^{2 g}}\right),
$$

the product being over all primes dividing $m$.

Let $\alpha$ be the involution on characteristic vectors mod 1 that sends $\left[\begin{array}{l}a \\ b\end{array}\right]$ to $\left[\begin{array}{l}-a \\ -b\end{array}\right]$, and for any set $S$ of characteristic vectors mod 1 upon which $\alpha$ acts, let $S / \alpha$ denote the quotient set of $S$ modulo the action of $\alpha$.

Lemma 1. (i) For $m$ odd, $\delta$ a theta characteristic, and $\gamma \in \Gamma(2)$, the symplectic action of $\gamma$ on characteristic vectors mod 1 leaves $\operatorname{char}_{\delta}(m)$ stable. This induces an action of $\Gamma$ on the sets

$$
\operatorname{even}(m)=\bigcup_{\delta \text { even }} \operatorname{char}_{\delta}(m), \quad \operatorname{odd}(m)=\bigcup_{\delta \text { odd }} \operatorname{char}_{\delta}(m) .
$$

(ii) For $m$ a multiple of $4, \operatorname{char}_{\delta}(m)=\operatorname{prim}(m)$ for all theta characteristics $\delta$. Furthermore, the symplectic action on characteristic vectors mod 1 gives an action of $\Gamma$ on $\operatorname{prim}(m)$.

(iii) The symplectic action on characteristic vectors mod 1 induces an action of $\Gamma(2)$ on $\operatorname{char}_{\delta}(m) / \alpha$. This induces actions of $\Gamma$ on even $(m) / \alpha$ and $\operatorname{odd}(m) / \alpha$ when $m$ is odd, and on $\operatorname{prim}(m)$ when $m$ is a multiple of 4 .

REMARK. For $m=2 m^{\prime}, m^{\prime}$ odd, we have $\operatorname{prim}(m)=\bigcup_{\delta \neq\left[\begin{array}{l}0 \\ 0\end{array}\right]} \operatorname{char}_{\delta}\left(m^{\prime}\right)$, the union being over all non-zero theta characteristics.

Proof. (i) Suppose $m$ is odd, $\left[\begin{array}{l}a \\ b\end{array}\right] \in \operatorname{char}_{\delta}(m)$, so

$$
\left[\begin{array}{l}
a \\
b
\end{array}\right]=\delta+\left[\begin{array}{l}
c \\
d
\end{array}\right] \bmod 1
$$


for some $\left[\begin{array}{l}c \\ d\end{array}\right] \in \operatorname{prim}(m)$. The symplectic action of $\gamma \in \Gamma=\left(\begin{array}{ll}A & B \\ C & D\end{array}\right)$ on $\left[\begin{array}{l}a \\ b\end{array}\right]$ from (5) is such that

$$
\left[\begin{array}{l}
a \\
b
\end{array}\right]^{\gamma}=\delta^{\gamma}+\left(\begin{array}{cc}
D & -C \\
-B & A
\end{array}\right)\left[\begin{array}{l}
c \\
d
\end{array}\right] \bmod 1
$$

It is easy to check that the symplectic action of $\Gamma(2)$ fixes $\delta \bmod 1$, and that the multiplicative action on characteristic vectors mod 1 defined by

$$
\left[\begin{array}{l}
c \\
d
\end{array}\right] \rightarrow\left(\begin{array}{cc}
D & -C \\
-B & A
\end{array}\right)\left[\begin{array}{l}
c \\
d
\end{array}\right] \bmod 1
$$

is an action of $\Gamma$ on $\operatorname{prim}(m)$. Therefore the symplectic action of $\Gamma(2)$ on characteristic vectors mod 1 defines an action on $\operatorname{char}_{\delta}(m)$. Hence the symplectic action of $\Gamma$ on characteristic vectors mod 1 defines actions on the sets even $(m)$ and $\operatorname{odd}(m)$.

(ii) For $m$ a multiple of 4 , and $\delta$ a theta characteristic, it is clear that $\operatorname{char}_{\delta}(m)=\operatorname{prim}(m)$. For $\gamma=\left(\begin{array}{ll}A & B \\ C & D\end{array}\right) \in \Gamma$, the multiplicative action on characteristic vectors mod 1 permutes $\operatorname{prim}(m)$, and differs from the symplectic action by the addition of an element in $\frac{1}{2} \mathbb{Z}^{g} / \mathbb{Z}^{g}$. But $\operatorname{prim}(m)$ is invariant under the addition of such elements.

(iii) We have $\alpha(\delta)=\delta$ for any theta characteristic $\delta$, so $\alpha$ acts on $\operatorname{char}_{\delta}(m)$. It follows as in the proof of (i) that the symplectic action of $\Gamma(2)$ commutes with $\alpha$, so gives an action on $\operatorname{char}_{\delta}(m) / \alpha$. The rest follows as in the proofs of (i) and (ii).

Let $\operatorname{Prim}(m)$ and $\operatorname{Char}_{\delta}(m)$ denote respectively sets of representatives for the classes of characteristic vectors $\bmod 1$ in $\operatorname{prim}(m)$ and $\operatorname{char}_{\delta}(m)$. Let $\operatorname{Prim}(m) / \alpha$ and $\operatorname{Char}_{\delta}(m) / \alpha$ denote respectively sets of representatives for the classes of characteristic vectors mod 1 and modulo $\alpha$ in $\operatorname{prim}(m) / \alpha$ and $\operatorname{char}_{\delta}(m) / \alpha$.

Proposition 1. Let $m$ be any positive integer.

(i) For any theta characteristic $\delta$, and any $\left[\begin{array}{l}a \\ b\end{array}\right] \in \operatorname{Char}_{\delta}(m)$,

$$
\theta\left[\begin{array}{l}
a \\
b
\end{array}\right](0, \tau)^{4 m} \in M_{2 m}(\Gamma(2 m))
$$

and is independent of the choice of $\left[\begin{array}{l}a \\ b\end{array}\right] \bmod 1$.

(ii) For any theta characteristic $\delta$, and $m$ odd, if

$$
\phi_{\delta, m}(\tau)=\prod_{\left[\begin{array}{c}
a \\
b
\end{array}\right] \in \operatorname{Char}_{\delta}(m)} \theta\left[\begin{array}{l}
a \\
b
\end{array}\right](0, \tau),
$$


then $\left(\phi_{\delta, m}(\tau)\right)^{4 m}$ is a modular form of level 2 . For $m$ a multiple of 4 , if

$$
\phi_{m}(\tau)=\prod_{\left[\begin{array}{l}
a \\
b
\end{array}\right] \in \operatorname{Prim}(m)} \theta\left[\begin{array}{l}
a \\
b
\end{array}\right](0, \tau),
$$

then $\left(\phi_{m}(\tau)\right)^{4 m}$ is a modular form of level 1 .

(iii) For $m$ odd, if

$$
\phi_{\text {even }, m}(\tau)=\prod_{\delta \text { even }} \phi_{\delta, m}(\tau) \quad \text { and } \quad \phi_{\text {odd }, m}(\tau)=\prod_{\delta \text { odd }} \phi_{\delta, m}(\tau),
$$

then $\left(\phi_{\text {odd }, m}(\tau)\right)^{4 m}$ and $\left(\phi_{\text {even }, m}(\tau)\right)^{4 m}$ are modular forms of level 1 .

(iv) If $m$ is odd, take

$$
\begin{gathered}
f(\tau)=\psi_{\text {even }, m}(\tau)=\prod_{\delta \text { even }\left[\begin{array}{l}
a \\
b
\end{array}\right] \in \operatorname{Char}_{\delta}(m) / \alpha} \prod_{\delta} \theta\left[\begin{array}{l}
a \\
b
\end{array}\right](0, \tau), \quad \text { or } \\
f(\tau)=\psi_{\text {odd }, m}(\tau)=\prod_{\delta \text { odd }\left[\begin{array}{l}
a \\
b
\end{array}\right] \in \operatorname{Char}_{\delta}(m) / \alpha} \theta\left[\begin{array}{l}
a \\
b
\end{array}\right](0, \tau) .
\end{gathered}
$$

If $m$ is a multiple of 4 take

$$
f(\tau)=\psi_{m}(\tau)=\prod_{\left[\begin{array}{l}
a \\
b
\end{array}\right] \in \operatorname{Prim}(m) / \alpha} \theta\left[\begin{array}{l}
a \\
b
\end{array}\right](0, \tau) .
$$

- If $g=1$ and $m \geq 3$, then $f(\tau)^{\operatorname{gcd}(8 m, 12)}$ is a modular form of level 1 .

- If $g=m=1$, then $f(\tau)^{8}$ is a modular form of level 1 .

- If $g=2$, then $f(\tau)^{2}$ is a modular form of level 1 .

- If $g \geq 3$, then $f(\tau)$ is a modular form of level 1 .

Proof. (i) Since $\Gamma(2 m) \subset \Gamma(2)$, for $\gamma \in \Gamma(2 m), \varrho(\gamma)^{4}=1$. It is easy to verify then that $\zeta(\gamma)^{4 m}=1$. Further, $\left[\begin{array}{l}a \\ b\end{array}\right]^{\gamma} \equiv\left[\begin{array}{l}a \\ b\end{array}\right] \bmod 1$, so by (3) and (4),

$$
j_{\gamma}(\tau)^{2 m} \theta\left[\begin{array}{l}
a \\
b
\end{array}\right](0, \tau)^{4 m}=\left(\theta\left[\begin{array}{l}
a \\
b
\end{array}\right]^{\gamma}(0, \gamma \circ \tau)\right)^{4 m}=\left(\theta\left[\begin{array}{l}
a \\
b
\end{array}\right](0, \gamma \circ \tau)\right)^{4 m},
$$

and by $(3), \theta\left[\begin{array}{l}a \\ b\end{array}\right](0, \tau)^{4 m}$ depends only on $\left[\begin{array}{l}a \\ b\end{array}\right] \bmod 1$.

(ii) By Lemma 1, and part (i), this is just the product of modular forms which are permuted under the action of $\Gamma(2) / \Gamma(2 m)$ (or $\Gamma / \Gamma(2 m)$ for $m$ a multiple of 4$)$, where the action is $f(\tau) \mapsto f(\gamma \circ \tau) / j_{\gamma}(\tau)^{2 m}$. This drops the level to 2 for $m$ odd, and to 1 for $m$ a multiple of 4 .

(iii) For $m$ odd, as in (ii), this is just the product under the action of $\Gamma / \Gamma(2)$ of modular forms on $\Gamma(2)$, which drops the level to 1 .

(iv) If $g(\tau)^{n}$ is a modular form of weight $n k, k$ an integer, then the map $\gamma \mapsto g(\gamma \circ \tau) /\left(g(\tau) j_{\gamma}(\tau)^{k}\right)$ is a character on $\Gamma$. Let $m$ be odd or a multiple of 4 . 
Since $\theta\left[\begin{array}{l}-a \\ -b\end{array}\right](0, \tau)=\theta\left[\begin{array}{l}a \\ b\end{array}\right](0, \tau)$ for $m \geq 3, f(\tau)^{2}$ differs by at most a multiplicative constant from $\phi_{\text {odd }, m}(\tau), \phi_{\text {even }, m}(\tau)$, or $\phi_{m}(\tau)$. So for $m \geq 3$, since it is easy to check that $\sigma_{2 g}(m)$ is a multiple of 4 , we see from (4) that $f(\tau)^{8 m}$ is a modular form whose weight is divisible by $8 m$. It is known ([M, p. 169]) that the number of even and odd theta characteristics is $2^{g-1}\left(2^{g}+1\right)$ and $2^{g-1}\left(2^{g}-1\right)$, respectively. Hence when $m=1$, since $\alpha$ pointwise fixes theta characteristics, $f(\tau)$ differs by at most a multiplicative constant from $\phi_{\text {odd }, 1}(\tau)$ or $\phi_{\text {even, } 1}(\tau)$, so if $g>1, f(\tau)^{4}$ is a modular form whose weight is divisible by 4 .

So in any case, unless $g=m=1$, we find that $f(\tau)$ is a modular form with character on $\Gamma$. For $g=2$ every character of $\Gamma$ is of order dividing 2 ; and for $g \geq 3$ there are no non-trivial characters of $\Gamma$ ([K, pp. 43-44]). For $g=1$, [Leh, p. 349] shows that for $m \geq 3, f(\tau)$ times some power of $\eta(\tau)^{2}$ is a modular form, so $f(\tau)^{12}$ is a modular form.

Finally, if $g=m=1, \psi_{\text {odd }, 1}(\tau)=0$ and Lemma $2(\mathrm{i})$ below shows that $\psi_{\text {even, } 1}(\tau)$ is a constant multiple of $\eta(\tau)^{3}$, so $\psi_{\text {even, } 1}(\tau)^{8}$ is a modular form.

Remark. We will see in Proposition 2 that if $g=1$, and $c(f)$ is the number of theta functions in the product defining $f(\tau)$ in Proposition 1(iv), then $f(\tau)$ is a constant times $\eta(\tau)^{c(f)}$.

2. Theta functions in one variable. Here $\Gamma=\mathrm{SL}_{2}(\mathbb{Z})$. The only odd theta characteristic is represented by $\left[\begin{array}{l}1 / 2 \\ 1 / 2\end{array}\right]$. We take $\left[\begin{array}{l}0 \\ 0\end{array}\right],\left[\begin{array}{c}0 \\ 1 / 2\end{array}\right]$, and $\left[\begin{array}{c}1 / 2 \\ 0\end{array}\right]$ as representatives for the three even theta characteristics. We recall some classic facts about modular forms of degree 1 (see, e.g. [M]). For $\tau \in \mathfrak{h}=\mathfrak{h}_{1}$, set $q=e^{2 \pi i \tau}$. For any modular form, its " $q$-expansion" is its Fourier series at " $i \infty$ " in $q$. If $f(\tau)$ is holomorphic on $\mathfrak{h}$ and $f(\tau)^{n}$ is a modular form, then $f(\tau)$ has a $q$-expansion in $q^{1 / n}=e^{2 \pi i \tau / n}$. The exponent of $q$ in the lead term of the $q$-expansion is the order of zero of a form at $i \infty$. Recall we define

$$
\eta(\tau)=q^{1 / 24} \prod_{n>0}\left(1-q^{n}\right) \quad \text { and } \quad \Delta(\tau)=\eta(\tau)^{24}
$$

We let $\theta^{\prime}\left[\begin{array}{l}a \\ b\end{array}\right](z, \tau)$ denote $\frac{d}{d z} \theta\left[\begin{array}{l}a \\ b\end{array}\right](z, \tau)$.

LEMMA 2. (i) We have

$$
\frac{1}{2 \pi i} \theta^{\prime}\left[\begin{array}{l}
1 / 2 \\
1 / 2
\end{array}\right](0, \tau)=\frac{i}{2} \theta\left[\begin{array}{l}
0 \\
0
\end{array}\right](0, \tau) \theta\left[\begin{array}{c}
1 / 2 \\
0
\end{array}\right](0, \tau) \theta\left[\begin{array}{c}
0 \\
1 / 2
\end{array}\right](0, \tau)=i \eta^{3}(\tau) \text {. }
$$

(ii) $\Delta(\tau)$ is a modular form of level 1 and weight 12 . It has no zeros on $\mathfrak{h}$ and a simple zero at $i \infty$.

(iii) The only modular forms of any level which have weight 0 are constants. 
Proof. See [M, p. 42 and pp. 64-72]. (i) is Jacobi's derivative formula. For any positive integer $m$, we define

$$
\begin{aligned}
& \operatorname{prod}\left[\begin{array}{l}
1 / 2 \\
1 / 2
\end{array}\right](m)(\tau)=\prod_{\substack{0 \leq u, v<m \\
(u, v) \neq(0,0)}} \theta\left[\begin{array}{c}
1 / 2+u / m \\
1 / 2+v / m
\end{array}\right](0, \tau), \\
& \operatorname{prod}\left[\begin{array}{l}
0 \\
0
\end{array}\right](m)(\tau)=\prod_{\substack{0 \leq u, v<m \\
(u, v) \neq(m / 2, m / 2), m \text { even } \\
(u, v) \neq(0,0), m \text { odd }}} \theta\left[\begin{array}{l}
u / m \\
v / m
\end{array}\right](0, \tau), \\
& \operatorname{prod}\left[\begin{array}{c}
1 / 2 \\
0
\end{array}\right](m)(\tau)=\prod_{\substack{0 \leq u, v<m \\
(u, v) \neq(0, m / 2), m \text { even } \\
(u, v) \neq(0,0), m \text { odd }}} \theta\left[\begin{array}{c}
1 / 2+u / m \\
v / m
\end{array}\right](0, \tau), \\
& \left.\operatorname{prod}\left[\begin{array}{c}
0 \\
1 / 2
\end{array}\right](m)(\tau)=\underset{\substack{0 \leq u, v<m \\
(u, v) \neq(m / 2,0), m \text { even } \\
(u, v) \neq(0,0), m \text { odd }}}{1 / 2+v / m}\right](0, \tau) .
\end{aligned}
$$

LEMMA 3. The following are lead terms of q-expansions:

$$
m \text { odd }
$$

$\operatorname{prod}\left[\begin{array}{l}1 / 2 \\ 1 / 2\end{array}\right](m)(\tau): \quad m q^{\left(m^{2}-1\right) / 24}$

$\operatorname{prod}\left[\begin{array}{l}0 \\ 0\end{array}\right](m)(\tau): \quad q^{\left(m^{2}-1\right) / 24}$

$\operatorname{prod}\left[\begin{array}{c}1 / 2 \\ 0\end{array}\right](m)(\tau): \quad(-1)^{(m-1) / 2} q^{\left(m^{2}-1\right) / 24}$

$\operatorname{prod}\left[\begin{array}{c}0 \\ 1 / 2\end{array}\right](m)(\tau): \quad q^{\left(m^{2}-1\right) / 24}$ meven

$$
\begin{aligned}
& -m q^{\left(m^{2}-1\right) / 24} \\
& (-1)^{(m-2) / 2} m q^{\left(m^{2}-1\right) / 24} \\
& (-1)^{(m-2) / 2} m q^{\left(m^{2}-1\right) / 24} \\
& -m q^{\left(m^{2}-1\right) / 24}
\end{aligned}
$$

Proof. For any theta function $\theta\left[\begin{array}{l}a \\ b\end{array}\right](0, \tau)$ with $a, b \in \mathbb{Q}$, we can compute its $q$-expansion directly from its definition (2). Alternatively, one can use the product expansion for theta functions (see [M, p. 69]). We leave the verification of the lemma to the reader.

Proposition 2.

$$
\text { modd } \quad \text { meven }
$$

$$
\begin{aligned}
& \operatorname{prod}\left[\begin{array}{l}
1 / 2 \\
1 / 2
\end{array}\right](m)(\tau)=\quad m \eta(\tau)^{m^{2}-1} \quad-m \eta(\tau)^{m^{2}-1} \\
& \operatorname{prod}\left[\begin{array}{l}
0 \\
0
\end{array}\right](m)(\tau)=\quad \eta(\tau)^{m^{2}-1} \quad(-1)^{(m-2) / 2} m \eta(\tau)^{m^{2}-1}
\end{aligned}
$$




$$
\begin{array}{ll}
\operatorname{prod}\left[\begin{array}{c}
1 / 2 \\
0
\end{array}\right](m)(\tau)=(-1)^{(m-1) / 2} \eta(\tau)^{m^{2}-1} & (-1)^{(m-2) / 2} m \eta(\tau)^{m^{2}-1} \\
\operatorname{prod}\left[\begin{array}{c}
0 \\
1 / 2
\end{array}\right](m)(\tau)=\eta(\tau)^{m^{2}-1} & -m \eta(\tau)^{m^{2}-1}
\end{array}
$$

Proof. First let us consider prod $\left[\begin{array}{l}1 / 2 \\ 1 / 2\end{array}\right](m)(\tau)$. Since $\left[\begin{array}{l}1 / 2 \\ 1 / 2\end{array}\right]$ represents the only odd theta characteristic when $g=1$, applying Proposition 1 to all the factors on the right hand side of

$$
\begin{aligned}
& \left(\operatorname{prod}\left[\begin{array}{l}
1 / 2 \\
1 / 2
\end{array}\right](m)(\tau)\right)^{4 m} \\
& \quad=\left(\prod_{d|m, 4| d} \phi_{d}(\tau) \cdot \prod_{2 d^{\prime} \mid m, d^{\prime} \text { odd }} \phi_{\text {even }, d^{\prime}}(\tau) \cdot \prod_{d \mid m, d>1 \text { odd }} \phi_{\text {odd }, d}(\tau)\right)^{4 m},
\end{aligned}
$$

we see that $\left(\operatorname{prod}\left[\begin{array}{l}1 / 2 \\ 1 / 2\end{array}\right](m)(\tau)\right)^{4 m}$ is a modular form of level 1 , and weight $2 m\left(m^{2}-1\right)$. By Lemma 3 , the lead term of its $q$-expansion is a constant times $q^{m\left(m^{2}-1\right) / 6}$. Therefore, by Lemma 2 ,

$$
\frac{\left(\operatorname{prod}\left[\begin{array}{l}
1 / 2 \\
1 / 2
\end{array}\right](m)(\tau)\right)^{4 m}}{\Delta(\tau)^{m\left(m^{2}-1\right) / 6}}
$$

is a modular form of level 1 and weight 0 , and hence a constant. Since $\mathfrak{h}$ is connected, prod $\left[\begin{array}{l}1 / 2 \\ 1 / 2\end{array}\right](m)(\tau)$ and $\eta(\tau)^{m^{2}-1}$ differ only by a constant. The constant is determined by the $q$-expansion in Lemma 3.

For any $\delta=\left[\begin{array}{l}0 \\ 0\end{array}\right],\left[\begin{array}{c}0 \\ 1 / 2\end{array}\right],\left[\begin{array}{c}1 / 2 \\ 0\end{array}\right]$, all of which represent even theta characteristics, the same argument only shows, a priori, that $(\operatorname{prod}[\delta](m)(\tau))^{4 m}$ is of level 2. But since each image under the action of $\Gamma$ has a $q$-expansion whose lead term is a constant times $q^{m\left(m^{2}-1\right) / 6}$, we deduce again that $(\operatorname{prod}[\delta](m)(\tau))^{4 m} / \Delta(\tau)^{m\left(m^{2}-1\right) / 6}$ is a modular form of level 2 and weight 0 , and hence a constant. Therefore, again $\operatorname{prod}[\delta](m)(\tau)$ and $\eta(\tau)^{m^{2}-1}$ differ only by a constant, determined by the $q$-expansions in Lemma 3 .

Proposition 3. Let $m$ be any positive integer.

(i) For any theta characteristic $\delta$, and any $\left[\begin{array}{l}a \\ b\end{array}\right] \in \operatorname{Char}_{\delta}(m)$,

$$
\theta^{\prime}\left[\begin{array}{l}
a \\
b
\end{array}\right](0, \tau)^{4 m} \in M_{6 m}(\Gamma(2 m)),
$$

and is independent of the choice of $\left[\begin{array}{l}a \\ b\end{array}\right] \bmod 1$. 
(ii) For any theta characteristic $\delta$, and $m$ odd, if

$$
\Phi_{\delta, m}(\tau)=\prod_{\left[\begin{array}{c}
a \\
b
\end{array}\right] \in \operatorname{Char}_{\delta}(m)} \theta^{\prime}\left[\begin{array}{l}
a \\
b
\end{array}\right](0, \tau),
$$

then $\left(\Phi_{\delta, m}(\tau)\right)^{4 m}$ is a modular form of level 2 . For $m$ a multiple of 4 , if

$$
\Phi_{m}(\tau)=\prod_{\left[\begin{array}{c}
a \\
b
\end{array}\right] \in \operatorname{Prim}(m)} \theta^{\prime}\left[\begin{array}{l}
a \\
b
\end{array}\right](0, \tau)
$$

then $\left(\Phi_{m}(\tau)\right)^{4 m}$ is a modular form of level 1 .

(iii) For $m$ odd, if

$$
\Phi_{\text {even }, m}(\tau)=\prod_{\delta \text { even }} \Phi_{\delta, m}(\tau) \quad \text { and } \quad \Phi_{\text {odd }, m}(\tau)=\Phi_{\delta, m}(\tau)
$$

for $\delta=\left[\begin{array}{l}1 / 2 \\ 1 / 2\end{array}\right] \bmod 1$, then $\left(\Phi_{\text {odd }, m}(\tau)\right)^{4 m}$ and $\left(\Phi_{\text {even }, m}(\tau)\right)^{4 m}$ are modular forms of level 1.

(iv) If $m$ is odd, take

$$
\begin{aligned}
& F(\tau)=\Psi_{\text {even }, m}(\tau)=\prod_{\delta \text { even }\left[\begin{array}{l}
a \\
b
\end{array}\right] \in \operatorname{Char}_{\delta}(m) / \alpha} \theta^{\prime}\left[\begin{array}{l}
a \\
b
\end{array}\right](0, \tau), \quad \text { or } \\
& F(\tau)=\Psi_{\text {odd }, m}(\tau)=\prod_{\left[\begin{array}{c}
a \\
b
\end{array}\right] \in \operatorname{Char}_{\delta}(m) / \alpha} \theta^{\prime}\left[\begin{array}{l}
a \\
b
\end{array}\right](0, \tau)
\end{aligned}
$$

for $\delta=\left[\begin{array}{l}1 / 2 \\ 1 / 2\end{array}\right] \bmod 1$. If $m$ is a multiple of 4 , take

$$
F(\tau)=\Psi_{m}(\tau)=\prod_{\left[\begin{array}{c}
a \\
b
\end{array}\right] \in \operatorname{Prim}(m) / \alpha} \theta^{\prime}\left[\begin{array}{l}
a \\
b
\end{array}\right](0, \tau) .
$$

Then if $m \geq 3, F(\tau)$ times some power of $\eta(\tau)^{2}$ is a modular form of level 1, so $F(\tau)^{\operatorname{gcd}(8 m, 12)}$ is a modular form of level 1 . If $m=1, F(\tau)^{8}$ is a modular form of level 1 .

Proof. These follow just as Proposition 1 by differentiating (4) and using the resulting formula at $z=0$.

Unlike the products in Proposition 2, the products $F(\tau)$ in Proposition 3(iv) are not necessarily constants times a power of $\eta(\tau)$. However, we will show in Theorem 1 that this is true for $m=3$ and $m=4$. For an analysis of these products for all $m$, see [BG].

For any representative $\left[\begin{array}{l}a \\ b\end{array}\right]$ of a theta characteristic, we let

$$
\operatorname{derivprod}\left[\begin{array}{l}
a \\
b
\end{array}\right](3)(\tau)=\prod_{\substack{0 \leq u, v<3 \\
(u, v) \neq(0,0)}} \theta^{\prime}\left[\begin{array}{l}
a+u / 3 \\
b+v / 3
\end{array}\right](0, \tau)
$$


and set

$$
\operatorname{derivprod}(4)(\tau)=\prod_{\substack{0 \leq u, v<4 \\
(u, v) \neq(0,0),(0,2),(2,0),(2,2)}} \theta^{\prime}\left[\begin{array}{l}
u / 4 \\
v / 4
\end{array}\right](0, \tau) .
$$

Part (ii) of the following theorem can be considered a generalization of Jacobi's derivative formula (Lemma 2(i)).

Theorem 1. For $\left[\begin{array}{l}a \\ b\end{array}\right]=\left[\begin{array}{l}0 \\ 0\end{array}\right],\left[\begin{array}{c}0 \\ 1 / 2\end{array}\right],\left[\begin{array}{c}1 / 2 \\ 0\end{array}\right]$ : is

(i) The lead term of the q-expansions of $\theta\left[\begin{array}{l}a \\ b\end{array}\right](0, \tau)^{8} \operatorname{derivprod}\left[\begin{array}{l}a \\ b\end{array}\right](3)(\tau)$

$$
\frac{(-1)^{2 a+1} 2^{8} \pi^{8}}{3^{5}} \cdot q^{4 / 3}
$$

The lead term of the q-expansion of derivprod $(4)(\tau)$ is $\left(-\pi^{12} / 2^{3}\right) q^{3 / 2}$.

(ii)

$$
\theta\left[\begin{array}{l}
a \\
b
\end{array}\right](0, \tau)^{8} \text { derivprod }\left[\begin{array}{l}
a \\
b
\end{array}\right](3)(\tau)=\frac{(-1)^{2 a+1} 2^{8} \pi^{8}}{3^{5}} \eta(\tau)^{32}
$$

and

$$
\operatorname{derivprod}(4)(\tau)=\frac{-\pi^{12}}{2^{3}} \eta(\tau)^{36} .
$$

Proof. (i) This is a computation whose verification we leave to the reader.

(ii) This is entirely similar to the proof of Proposition 2.

3. Theta functions in two variables. Here $\Gamma=\mathrm{Sp}_{4}(\mathbb{Z})$. The structure of the ring $\bigcup_{k \geq 0} M_{k}(\Gamma)$ was determined by Igusa [I1] and subsequently by Hammond $[\mathrm{H}]$, and Freitag $[\mathrm{F}]$.

There are six odd theta characteristics, represented by

$$
\left[\begin{array}{c}
1 / 2 \\
0 \\
1 / 2 \\
0
\end{array}\right], \quad\left[\begin{array}{c}
1 / 2 \\
1 / 2 \\
1 / 2 \\
0
\end{array}\right],\left[\begin{array}{c}
1 / 2 \\
0 \\
1 / 2 \\
1 / 2
\end{array}\right],\left[\begin{array}{c}
0 \\
1 / 2 \\
0 \\
1 / 2
\end{array}\right],\left[\begin{array}{c}
1 / 2 \\
1 / 2 \\
0 \\
1 / 2
\end{array}\right], \quad\left[\begin{array}{c}
0 \\
1 / 2 \\
1 / 2 \\
1 / 2
\end{array}\right] .
$$

Representatives for the 10 even theta characteristics are

$$
\begin{gathered}
{\left[\begin{array}{c}
0 \\
0 \\
0 \\
0
\end{array}\right], \quad\left[\begin{array}{c}
1 / 2 \\
0 \\
0 \\
0
\end{array}\right], \quad\left[\begin{array}{c}
1 / 2 \\
1 / 2 \\
0 \\
0
\end{array}\right], \quad\left[\begin{array}{c}
0 \\
1 / 2 \\
0 \\
0
\end{array}\right],\left[\begin{array}{c}
1 / 2 \\
0 \\
0 \\
1 / 2
\end{array}\right],} \\
{\left[\begin{array}{c}
0 \\
1 / 2 \\
1 / 2 \\
0
\end{array}\right], \quad\left[\begin{array}{c}
0 \\
0 \\
1 / 2 \\
0
\end{array}\right],\left[\begin{array}{c}
0 \\
0 \\
1 / 2 \\
1 / 2
\end{array}\right],\left[\begin{array}{c}
0 \\
0 \\
0 \\
1 / 2
\end{array}\right],\left[\begin{array}{c}
1 / 2 \\
1 / 2 \\
1 / 2 \\
1 / 2
\end{array}\right] .}
\end{gathered}
$$


We write

$$
\tau=\left(\begin{array}{ll}
\tau_{11} & \tau_{12} \\
\tau_{12} & \tau_{22}
\end{array}\right) \quad \text { for } \tau \in \mathfrak{h}_{2}
$$

Let $Z$ denote the image of the subvariety $\tau_{12}=0$ of $\mathfrak{h}_{2}$ under the action by $\Gamma$.

We define

$$
\Delta_{2}(\tau)=2^{-12} \prod_{\delta \text { even }} \theta[\delta]^{2}(0, \tau)
$$

We need to accumulate some facts.

LEMMA 4. (i) $\Delta_{2}(\tau)$ is a modular form of level 1 and weight 10, which has zeros of order 2 along $Z$ and no other zeros.

(ii) A modular form of any level which is of weight 0 is a constant.

Proof. These can be found in [K, pp. 115, 119].

We call $\Delta_{2}(\tau)$ the discriminant modular form (of degree 2). The reason for the name is that via Thomae's formula, it can be shown for $\tau \notin Z$ that $\Delta_{2}(\tau)$ differs only by a multiplicative constant from the discriminant of the curve of genus 2 whose period matrix is $\tau$ (see [Gr3]).

It follows from the definition (2) that if $\tau=\left(\begin{array}{ll}\tau_{11} & \tau_{12} \\ \tau_{12} & \tau_{22}\end{array}\right) \in \mathfrak{h}_{2}$, then for $a=\left[\begin{array}{l}a_{1} \\ a_{2}\end{array}\right], b=\left[\begin{array}{l}b_{1} \\ b_{2}\end{array}\right], a_{i}, b_{i} \in \mathbb{R}, i=1,2$, we have

$$
\left.\theta\left[\begin{array}{l}
a \\
b
\end{array}\right](0, \tau)\right|_{\tau_{12}=0}=\theta\left[\begin{array}{l}
a_{1} \\
b_{1}
\end{array}\right]\left(0, \tau_{11}\right) \theta\left[\begin{array}{l}
a_{2} \\
b_{2}
\end{array}\right]\left(0, \tau_{22}\right)
$$

Recall that $\theta\left[\begin{array}{l}a_{i} \\ b_{i}\end{array}\right]\left(0, \tau_{i i}\right)=0$ if and only if $\left[\begin{array}{l}a_{i} \\ b_{i}\end{array}\right] \equiv\left[\begin{array}{l}1 / 2 \\ 1 / 2\end{array}\right] \bmod 1([\mathrm{M}, \mathrm{p} .11])$.

TheOREM 2. For any odd theta characteristic $\delta=\left[\begin{array}{l}a_{1} \\ a_{2} \\ b_{1} \\ b_{2}\end{array}\right]$,

$$
f_{\delta}(\tau):=\prod_{\substack{0 \leq u_{i}, v_{i}<3 \\
\left(u_{1}, u_{2}, v_{1}, v_{2}\right) \neq(0,0,0,0)}} \theta\left[\begin{array}{r}
u_{1} / 3 \\
u_{2} / 3 \\
v_{1} / 3 \\
v_{2} / 3
\end{array}\right](0, \tau)=c_{3}(\delta) \Delta_{2}(\tau)^{4}
$$

where $c_{3}(\delta)=(-1)^{2 a_{1}+2 a_{2}} 3^{4}$, and

$$
g(\tau):=\prod_{\substack{0 \leq u_{i}, v_{i}<4 \\
\left(u_{1}, u_{2}, v_{1}, v_{2}\right) \text { not all even }}} \theta\left[\begin{array}{c}
u_{1} / 4 \\
u_{2} / 4 \\
v_{1} / 4 \\
v_{2} / 4
\end{array}\right](0, \tau)=c_{4} \cdot \Delta_{2}(\tau)^{12}
$$

where $c_{4}=2^{24}$. 
Proof. By Proposition $1, f_{\delta}(\tau)^{12}$ is a modular form of level 2 and weight 480. Note that by (6), for every odd characteristic $\delta, 8$ terms in the product $f_{\delta}(\tau)$ vanish when $\tau_{12}=0$. Since the 6 choices of $f_{\delta}(\tau)^{12}$ are permuted by $\Gamma$, for each $\delta, f_{\delta}(\tau)^{12}$ vanishes at least to order 96 on $Z$. So by Lemma 4 , $f_{\delta}(\tau)^{12} /\left(\Delta_{2}(\tau)\right)^{48}$ is a modular form of level 2 and weight 0 , which is necessarily a constant. Therefore $f_{\delta}(\tau)^{12}$ differs by a constant from $\Delta_{2}(\tau)^{48}$, and since $\mathfrak{h}_{2}$ is connected, $f_{\delta}(\tau)$ differs by a constant from $\Delta_{2}(\tau)^{4}$. A calculation with (3) shows that the constant is independent of the choice of representative for $\delta \bmod 1$.

By Proposition 1, $g(\tau)^{2}$ is a modular form of weight 240 and level 1. Of the 240 terms in the product $g, 24$ vanish along $Z$. Therefore $g(\tau)^{2} / \Delta_{2}(\tau)^{24}$ is a modular form of weight 0 and level 1 , and hence a constant. Therefore $g(\tau)$ and $\Delta_{2}(\tau)^{12}$ differ by a constant.

It remains to compute $c_{3}(\delta)$ and $c_{4}$. For this we need to take the Taylor expansion of $\theta\left[\begin{array}{l}a \\ b\end{array}\right](0, \tau)$ in $\tau_{12}$ at 0 . If the function does not vanish on $\tau_{12}=0$, the lead term in the expansion is given by (6). If it does vanish, the lead term is given by $\tau_{12}$ times

$$
\left.\frac{d}{d \tau_{12}} \theta\left[\begin{array}{l}
a \\
b
\end{array}\right](0, \tau)\right|_{\tau_{12}=0}=\frac{1}{2 \pi i} \theta^{\prime}\left[\begin{array}{l}
a_{1} \\
b_{1}
\end{array}\right]\left(0, \tau_{11}\right) \theta^{\prime}\left[\begin{array}{l}
a_{2} \\
b_{2}
\end{array}\right]\left(0, \tau_{22}\right) .
$$

For starters, we compute the lead term of the Taylor expansion of $\Delta_{2}(\tau)$ as

$$
\begin{aligned}
2^{-12}(2 \pi i)^{2}( & \left.\frac{1}{2 \pi i} \theta^{\prime}\left[\begin{array}{l}
1 / 2 \\
1 / 2
\end{array}\right]\left(0, \tau_{11}\right)\right)^{2}\left(\frac{1}{2 \pi i} \theta^{\prime}\left[\begin{array}{l}
1 / 2 \\
1 / 2
\end{array}\right]\left(0, \tau_{22}\right)\right)^{2} \\
& \times\left(\theta\left[\begin{array}{l}
0 \\
0
\end{array}\right]\left(0, \tau_{11}\right) \theta\left[\begin{array}{c}
0 \\
1 / 2
\end{array}\right]\left(0, \tau_{11}\right) \theta\left[\begin{array}{c}
1 / 2 \\
0
\end{array}\right]\left(0, \tau_{11}\right)\right)^{6} \\
& \times\left(\theta\left[\begin{array}{l}
0 \\
0
\end{array}\right]\left(0, \tau_{22}\right) \theta\left[\begin{array}{c}
0 \\
1 / 2
\end{array}\right]\left(0, \tau_{22}\right) \theta\left[\begin{array}{c}
1 / 2 \\
0
\end{array}\right]\left(0, \tau_{22}\right)\right)^{6}\left(\tau_{12}\right)^{2} \\
= & -2^{2} \pi^{2} \Delta\left(\tau_{11}\right) \Delta\left(\tau_{22}\right)\left(\tau_{12}\right)^{2},
\end{aligned}
$$

by Lemma 2 .

The formulas in Section 2 now give enough ammunition to calculate $c_{3}(\delta)$ and $c_{4}$.

We will compute the Taylor expansion of $f_{\delta_{0}}(\tau)$ when $\delta_{0}=\left[\begin{array}{c}1 / 2 \\ 1 / 2 \\ 0 \\ 1 / 2\end{array}\right]$. The other choices for odd theta characteristics are treated similarly. The lead term of the expansion is

$$
\left(\frac{1}{2 \pi i} \theta^{\prime}\left[\begin{array}{l}
1 / 2 \\
1 / 2
\end{array}\right]\left(0, \tau_{22}\right)\right)^{8} \theta\left[\begin{array}{c}
1 / 2 \\
0
\end{array}\right]\left(0, \tau_{11}\right)^{8} \text { derivprod }\left[\begin{array}{c}
1 / 2 \\
0
\end{array}\right](3)\left(\tau_{11}\right)
$$




$$
\begin{aligned}
& \times\left(\operatorname{prod}\left[\begin{array}{l}
1 / 2 \\
1 / 2
\end{array}\right](3)\left(\tau_{22}\right)\right)^{9}\left(\operatorname{prod}\left[\begin{array}{c}
1 / 2 \\
0
\end{array}\right](3)\left(\tau_{11}\right)\right)^{8}\left(\tau_{12}\right)^{8} \\
= & 3^{4} 2^{8} \pi^{8} \Delta\left(\tau_{11}\right)^{4} \Delta\left(\tau_{22}\right)^{4}\left(\tau_{12}\right)^{8}
\end{aligned}
$$

so $c_{3}\left(\delta_{0}\right)=3^{4}$.

Finally, the lead term of the Taylor expansion of $g(\tau)$ is

$$
\begin{aligned}
\left(\frac{1}{2 \pi i} \theta^{\prime}\left[\begin{array}{l}
1 / 2 \\
1 / 2
\end{array}\right]\right. & \left.\left(0, \tau_{11}\right)\right)^{12}\left(\frac{1}{2 \pi i} \theta^{\prime}\left[\begin{array}{l}
1 / 2 \\
1 / 2
\end{array}\right]\left(0, \tau_{22}\right)\right)^{12} \\
& \times \operatorname{derivprod}(4)\left(\tau_{11}\right) \text { derivprod }(4)\left(\tau_{22}\right) \\
& \times\left(\theta\left[\begin{array}{l}
0 \\
0
\end{array}\right]\left(0, \tau_{11}\right) \theta\left[\begin{array}{c}
0 \\
1 / 2
\end{array}\right]\left(0, \tau_{11}\right) \theta\left[\begin{array}{c}
1 / 2 \\
0
\end{array}\right]\left(0, \tau_{11}\right)\right)^{-3} \\
& \times\left(\theta\left[\begin{array}{l}
0 \\
0
\end{array}\right]\left(0, \tau_{22}\right) \theta\left[\begin{array}{c}
0 \\
1 / 2
\end{array}\right]\left(0, \tau_{22}\right) \theta\left[\begin{array}{c}
1 / 2 \\
0
\end{array}\right]\left(0, \tau_{22}\right)\right)^{-3} \\
& \times\left(\operatorname{prod}\left[\begin{array}{l}
0 \\
0
\end{array}\right](4)\left(\tau_{11}\right)\right)^{15}\left(\operatorname{prod}\left[\begin{array}{c}
0 \\
0
\end{array}\right](4)\left(\tau_{22}\right)\right)^{15}\left(\tau_{12}\right)^{24} \\
= & 2^{48} \pi^{24} \Delta\left(\tau_{11}\right)^{12} \Delta\left(\tau_{22}\right)^{12}\left(\tau_{12}\right)^{24},
\end{aligned}
$$

so $c_{4}=2^{24}$.

Remark. For any $\tau \in \mathfrak{h}_{2}$ not in $Z, \tau$ is the period matrix of some complex curve $\mathcal{C}$ of genus 2 . The curve has six Weierstrass points, $w_{k}, 1 \leq$ $k \leq 6$, and the canonical divisor class is $2 w_{k}$ for any $k$. Fix one choice of $k$. We can pick a symplectic basis $A_{1}, A_{2}, B_{1}, B_{2}$ for $H_{1}(\mathcal{C}, \mathbb{Z})$ (i.e., such that $\left.A_{1} \cdot A_{2}=B_{1} \cdot B_{2}=0, A_{i} \cdot B_{j}=\delta_{i j}\right)$, and a normalized basis $\mu_{1}, \mu_{2}$ of holomorphic differentials of $\mathcal{C}$ such that

$$
\left[\int_{A_{i}} \mu_{j}\right]_{i, j=1,2}=I, \quad\left[\int_{B_{i}} \mu_{j}\right]_{i, j=1,2}=\tau .
$$

Then we have an embedding

$$
\mathcal{C} \stackrel{\Phi_{k}}{\longrightarrow} \mathbb{C}^{2} / L
$$

given by

$$
P \mapsto \int_{w_{k}}^{P}\left(\mu_{1}, \mu_{2}\right) \bmod L
$$

where $L$ is the lattice in $\mathbb{C}^{2}$ generated by the columns of $I$ and $\tau$. (For background and details, see [Gr5].) The map $\Phi_{k}$ extends by linearity to divisors of $\mathcal{C}$, and the Abel-Jacobi Theorem says that if $D$ is a divisor of degree 0 , then $D$ is the divisor of a function if and only if $\Phi_{k}(D)$ is the origin 
in $\mathbb{C}^{2} / L$. It follows that $\Phi_{k}\left(w_{j}\right), j=1, \ldots, 6$, are precisely the 2 -torsion points of $\mathbb{C}^{2} / L$ which lie on $\Phi_{k}(\mathcal{C})$.

A fundamental theorem of Riemann says that there is an odd theta characteristic $\delta=\delta(k)$ such that $\theta[\delta](z, \tau), z \in \mathbb{C}^{2}$, has a zero of order 1 along the pullback of $\Phi_{k}(\mathcal{C})$ to $\mathbb{C}^{2}$ and no other zeros. For $a, b \in \mathbb{R}^{2}$, since $\theta\left[\delta+{ }_{b}^{a}\right](0, \tau)$ differs by an exponential from $\theta[\delta](\tau a+b, \tau)$, we see that $\theta\left[\delta+{ }_{b}^{a}\right](0, \tau)=0$ if and only if $\tau a+b \in \Phi_{k}(\mathcal{C})$. Theorem 2 says $\theta\left[\delta+{ }^{c}\right](0, \tau) \neq 0$ for $\tau \notin Z$, when $3 c \equiv 3 d \equiv 0 \bmod 1$, and $c$ or $d \not \equiv 0 \bmod 1$, and that $\theta\left[\begin{array}{l}c \\ d\end{array}\right](0, \tau) \neq 0$ for $\tau \notin Z$ when $4 c \equiv 4 d \equiv 0 \bmod 1$, and $2 c$ or $2 d \not \equiv 0 \bmod 1$. With this we get

Corollary. There is no point $P$ on $\mathcal{C}, P \neq w_{k}$, such that $3\left(P-w_{k}\right)$ is the divisor of a function, and there is no point $P$ on $\mathcal{C}, P \neq w_{j}, 1 \leq j \leq 6$, such that $4\left(P-w_{k}\right)$ is the divisor of a function.

This corollary can easily be derived from the Riemann-Roch Theorem (see, e.g. [Box]). Having done so directly, Goren gave a moduli-theoretic proof of Theorem 2 up to an unspecified constant [Go]. Likewise, we can see that there is no analogue of Theorem 2 for $m=5$, because on the curve $\mathcal{C}: y^{2}=x^{5}+1$, the divisor of $y-1$ is $5((0,1)-\infty)$, where $\infty$ denotes the Weierstrass point at infinity (see $[\mathrm{BG}]$ ). See $[\mathrm{BGL}]$ for a complete description of the moduli space of curves of genus 2 such that there is a $P \in C, P \neq \infty$, such that $5(P-\infty)$ is the divisor of a function.

\section{References}

[A] J. Arledge, S-units attached to genus 3 hyperelliptic curves, J. Number Theory 63 (1997), 12-29.

[BaBo] E. Bavencoffe et J. Boxall, Valeurs des fonctions thêta associées à la courbe $y^{2}=x^{5}-1$, Séminaire de Théories des Nombres de Caen 1991/1992, 36 pp.

[Bor] R. Borcherds, Monstrous moonshine and monstrous Lie superalgebras, Invent. Math. 109 (1992), 405-444.

[Box] J. Boxall, Valeurs spéciales de fonctions abéliennes, Groupe de travail sur les problèmes diophantiens, Université de Paris VI, année 1990/1.

[BoBa] J. Boxall et E. Bavencoffe, Quelques propriétés arithmétiques des points de 3division de la jacobienne de $y^{2}=x^{5}-1$, Sém. Théor. Nombres Bordeaux 4 (1992), 113-128.

[BG] J. Boxall and D. Grant, Theta functions and singular torsion on elliptic curves, to appear in the proceedings of the Millenial number theory conference.

[BGL] J. Boxall, D. Grant and F. Leprévost, 5-torsion points on curves of genus 2, J. London Math. Soc. 64 (2001), 29-43.

[C] G. Coogan, A generalization of Jacobi's derivative formula, Ph.D. thesis, Univ. of Colorado at Boulder, 1999.

[F] E. Freitag, Zur Theorie der Modulformen zweiten Grades, Nachr. Akad. Wiss. Göttingen Math.-Phys. Kl. II 1965, 151-157. 
[FK] T. Fukuda and K. Komatsu, On a unit group generated by special values of Siegel modular functions, Math. Comp. 69 (2000), 1207-1212.

[Go] E. Goren, A note on vanishing properties of certain theta constants of genus two, Landau Center, preprint No. 15, 1995/96.

[Gr1] D. Grant, Units from 3- and 4-torsion on Jacobians of curves of genus 2, Compositio Math. 95 (1994), 311-320.

[Gr2] -, Units from 5-torsion on the Jacobian of $y^{2}=x^{5}+\frac{1}{4}$ and the conjectures of Stark and Rubin, J. Number Theory 77 (1999), 227-251.

[Gr3] - A generalization of Jacobi's derivative formula to dimension two, J. Reine Angew. Math. 392 (1988), 125-136.

[Gr4] - , Theta functions and division points on abelian varieties of dimension two, Ph.D. thesis, MIT, 1985.

[Gr5] - Formal groups in genus two, J. Reine Angew. Math. 411 (1990), 96-121.

$[\mathrm{H}] \quad$ W. Hammond, On the graded ring of Siegel modular forms of genus two, Amer. J. Math. 87 (1965), 502-506.

[I1] J. Igusa, On Siegel modular forms of genus two. I, II, ibid. 84 (1962), 175-200; 86 (1964), 392-412.

[I2] - Theta Functions, Springer, Berlin, 1972.

[K] H. Klingen, Introductory Lectures on Siegel Modular Forms, Cambridge Stud. Adv. Math. 20, Cambridge Univ. Press, Cambridge, 1990.

[Lec] O. Lecacheux, Unités de corps de nombres et courbes de genre un et deux, in: K. Dilcher (ed.), Number Theory (Halifax, NS, 1994), CMS Conf. Proc. 15, Amer. Math. Soc., 1995, 229-243.

[Leh] J. Lehner, Discontinuous Groups and Automorphic Functions, Math. Surveys Monographs 8, Amer. Math. Soc., Providence, 1964.

[M] D. Mumford, Tata Lectures on Theta. I, Progr. Math. 28, Birkhäuser, Boston, 1983.

[dSG] E. de Shalit and E. Goren, On special values of theta functions of genus two, Ann. Inst. Fourier (Grenoble) 47 (1997), 775-799.

[S] H. Stark, The Coates-Wiles theorem revisited, in: Number Theory Related to Fermat's Last Theorem, Progr. Math. 26, Birkhäuser, Boston, 1982, 349-362.

Department of Mathematics

University of Colorado at Boulder

Boulder, CO 80309-0395, U.S.A.

E-mail: grant@boulder.colorado.edu

Received on 27.7.1999

and in revised form on 22.6.2001 